\title{
DNA barcodes reveal female dimorphism in syringophilid mites (Actinotrichida: Prostigmata: Cheyletoidea): Stibarokris phoeniconaias and Ciconichenophilus phoeniconaias are conspecific
}

\author{
Eliza Glowska ${ }^{1}$, Anna Dragun-Damian $^{1}$, Lukasz Broda $^{1}$, Jacek Dabert $^{1}$ and Miroslawa Dabert $^{2}$ \\ ${ }^{1}$ Department of Animal Morphology, Faculty of Biology, Adam Mickiewicz University, Poznań, Poland; \\ ${ }^{2}$ Molecular Biology Techniques Laboratory, Faculty of Biology, Adam Mickiewicz University, Poznań, Poland
}

\begin{abstract}
Here we present the first evidence of female dimorphism in ectoparasitic quill mites of the family Syringophilidae (Actinotrichida: Prostigmata: Cheyletoidea). Stibarokris phoeniconaias Skoracki et OConnor, 2010 and Ciconichenophilus phoeniconaias Skoracki et OConnor, 2010 so far have been treated as two distinct species cohabiting inside the quills of feathers of the lesser flamingo Phoeniconaias minor (Geoffroy Saint-Hilaire) and the American flamingo Phoenicopterus ruber Linnaeus. Although females of these species differ morphologically by the extent of body sclerotisation, presence/absence of lateral hypostomal teeth, and shape of dorsal setae, their important common features are the lack of leg setae $v s I I$, and both stylophore and peritremes shape. Here, we apply the DNA barcode markers to test whether the differences between $S$. phoeniconaias and C. phoeniconaias have a genetic basis, indicating that they really are distinct taxa, or whether they just represent two morphs of a single species. All analysed sequences (616 bp for COI and $1159 \mathrm{bp}$ for 28S rDNA) obtained for specimens representing females of both studied taxa as well as male, tritonymph, protonymph and larva of S. phoeniconaias were identical, which indicates that S. phoeniconaias and C. phoeniconaias are conspecific. The formal taxonomic consequence of our results is denial of the genus status of Ciconichenophilus Skoracki et OConnor, 2010 and species status of C. phoeniconaias, and recommendation that they should be treated as junior synonyms of Stibarokris Kethley, 1970 and S. phoeniconaias, respectively.
\end{abstract}

Keywords: quill mites, Syringophilidae, DNA-barcoding, COI, 28S rDNA, systematics

Morphologically based systematic studies of mites (Arachnida: Actinotrichida and Anactinotrichida) may sometimes be misleading because of the phenomenon of di- and polymorphism, i.e. the case when two or more morphologically distinctive forms of the same sex occur within a single species. In males, this phenomenon was noticed, in Parasitidae (Dabert et al. 2011) and some cheyletid genera (Volgin 1969, Regev 1974) but most widespread and best studied was in Astigmata (Radwan 2001, Proctor et al. 2009), where is usually associated with various strategies for gaining access to females.

Female dimorphism has also been recorded in some groups of mites, e.g. in families Ascidae, Ascouracaridae, Dolichocybidae, Pygmephoridae and Scutacaridae. Although its meaning is not always fully understood, it is commonly associated with the presence of typical and migrating morphs or co-occurrence of a normal and overwintering forms (Cross and Moser 1975, Martin 1978, Magowski 1988, Lindquist and Walter 1989, Dabert and Ehrnsberger 1992, Manson and Oldfield 1996, Walter and Proctor 1999, Beaulieu et al. 2008).

The phenomenon of dimorphism can lead to erroneous results in taxonomic studies, particularly within mite groups with strongly unbalanced sex ratios. In such cases, new species descriptions are often based on one sex only, which causes a risk that morphs may be treated and described as separate taxa. One group in which the sex ratio is strongly unequal (female-biased) is the ectoparasitic quill mites family Syringophilidae (Actinotrichida: Prostigmata: Cheyletoidea). Within all described syringophilid species ( $\mathrm{ca} 300$ ), there exist many for which males have never been found (Skoracki 2011).

Strongly isolated microhabitats, such as a quill, are typically occupied by only one species. Within syringophilids, cases of co-inhabitation of a single quill by two different species are exceptional and so far have been restricted to the two cases. One involves females of Krantziaulonastus yoyomi Glowska et Skoracki, 2011 and Picobia dinemellia Glowska et Skoracki, 2011 in the body feather quills of the white-headed buffalo weaver Dinemellia dinemelli (Rüppell) (Glowska and Skoracki 2011a). The second case is the co-occurrence of Stibarokris phoeniconaias Skoracki et OConnor, 2010 and Ciconichenophilus phoeniconaias Skoracki et OConnor, 2010 inside the coverts and body feathers of the American flamingo Phoenicopterus ruber Linnaeus.

Address for correspondence: E. Glowska, Department of Animal Morphology, Faculty of Biology, A. Mickiewicz University, Umultowska 89, 61-614 Poznań, Poland. Phone: +48 61829 5685; Fax: +48 61829 5687; E-mail: glowska@amu.edu.pl 
Glowska et al.: Female dimorphism in syringophilid mites

The female of $S$. phoeniconaias was described from secondary coverts of the lesser flamingo Phoeniconaias minor (Geoffroy Saint-Hilaire) (Skoracki and OConnor 2010) and additionally reported from covert feathers of the American flamingo Phoenicopterus ruber (Glowska and Skoracki 2011b). The diagnosis of the species and genus were improved and supplemented by description of the male in Glowska and Skoracki (2011b) (first male record in the genus Stibarokris Kethley, 1970). Ciconichenophilus phoeniconaias was described from the quill of secondary feather of the lesser flamingo and assigned to the monotypic genus Ciconichenophilus Skoracki et OConnor, 2010. The male has never been found in this species (and therefore for this genus).

The co-occurrence of $S$. phoeniconaias and C. phoeniconaias in a single quill as two distinct species may be doubtful for three reasons: i) coexistence of two different species of syringophilids inside the same quill is a rare phenomenon; ii) the male of Ciconichenophilus has never been found; iii) although the females of $S$. phoeniconaias and $C$. phoeniconaias differ in degree of body sclerotisation, the presence/absence of lateral hypostomal teeth and dorsal setal morphology, they display also some important common features including lack of leg setae vs II, and both stylophore and peritreme shape. The S. phoeniconaias female actually looks like an 'armored' version of $C$. phoeniconaias. This prompted us to test the genetic distinction between these taxa.

Molecular methods have recently been applied to taxonomy of syringophilid mites both in extending the standard of new species description (Glowska et al. 2012a,b) and in verification of compatibility between morphological and molecular delimitations of species boundaries (Glowska et al. 2013). Here, we have applied DNA diagnostics (COI and 28S rDNA) to test whether the differences between females of Stibarokris phoeniconaias and Ciconichenophilus phoeniconaias have a genetic basis and they really are distinct taxa, or whether they are just two morphs of the same species.

\section{MATERIALS AND METHODS}

Animal material used in morphological and molecular analysis

Mites used in the study were collected from coverts of a frozen specimen of an American flamingo deposited in Biozentrum Grindel und Zoologisches Museum Hamburg at the University of Hamburg. Each covert was completely removed from the bird and dissected under an Olympus ZS30 stereomicroscope. Until DNA extraction, mites were preserved in $96 \%$ ethyl alcohol and, before mounting on microscopic slides, they were individually subjected to DNA extraction. Vouchers were mounted on slides in Faure medium. Photos and measurements were done by Olympus DP70 digital camera and Olympus BX51 light microscope with Nomarski differential interference contrast (DIC). All measurements, including scale bars in figures are given in micrometres $(\mu \mathrm{m})$. Latin names of birds follow Clements (2007). The voucher slides and corresponding DNA samples are deposited in the collection of the Department of Animal Mor-
Table 1. Animal material (specimens) used in the study.

\begin{tabular}{llccc}
\hline \multirow{2}{*}{ Species } & \multirow{2}{*}{ Sex/instar } & \multirow{2}{*}{ Voucher } & \multicolumn{2}{c}{ GenBank Acc. Nos. } \\
\cline { 4 - 5 } & & & COI & 28S rDNA \\
\hline Ciconichenophilus & Female & EG534 & KF840699 & KF840706 \\
phoeniconaias & Female & EG535 & KF840700 & KF840707 \\
Stibarokris & Female & EG615 & KF840705 & KF840708 \\
phoeniconaias & Female & EG618 & KF840704 & KF840709 \\
& Female & EG624 & KF840703 & KF840710 \\
& Male & EG657 & KF840701 & KF840711 \\
& Tritonymph & EG622 & KF840697 & KF840713 \\
& Protonymph & EG621 & KF840702 & KF840712 \\
& Larva & EG641 & KF840698 & KF840714 \\
\hline
\end{tabular}

phology, Faculty of Biology, Adam Mickiewicz University in Poznań, Poland, under the identification numbers indicated in Table 1.

\section{Molecular analysis}

Total genomic DNA was extracted from single specimens using DNeasy Blood \& Tissue Kit (Qiagen, Hilden, Germany) as described by Dabert et al. (2008). We used sequence data from the cytochrome oxidase subunit I (COI) gene fragment from the mitochondrial genome and a fragment comprising D1-D3 regions of the 28S rDNA as a representative of the nuclear DNA. The COI was amplified by PCR with degenerate primers: Aseq01F (GGAACRATATAYTTTATTTTTAGA) and Aseq03R (GGATCTCCWCCTCCWGATGGATT). PCR amplifications were carried out in $10 \mu \mathrm{l}$ reaction volumes containing $5 \mu \mathrm{l}$ of Type-it Microsatellite Kit (Qiagen), $0.5 \mu \mathrm{M}$ of each primer, and $4 \mu \mathrm{l}$ of DNA template using a thermocycling profile of one cycle of $5 \mathrm{~min}$ at $95^{\circ} \mathrm{C}$ followed by 35 steps of $30 \mathrm{~s}$ at $95^{\circ} \mathrm{C}, 1 \mathrm{~min}$ at $50^{\circ} \mathrm{C}, 1 \mathrm{~min}$ at $72^{\circ} \mathrm{C}$, with a final step of $5 \mathrm{~min}$ at $72^{\circ} \mathrm{C}$. Amplification of $28 \mathrm{~S}$ rDNA fragments was done with primers 28F0001 (ACCCVCYNAATTTAAGCATAT) and 28R0990 (CCTTGGTCCGTGTTTCAAGAC) (Mironov et al. 2012).

After amplification PCR products were two-fold diluted with water and $5 \mu 1$ of the sample was analysed by electrophoresis on a $1.0 \%$ agarose gel. Samples containing visible bands were directly sequenced using $1 \mu \mathrm{l}$ of the PCR reaction and $50 \mathrm{pmol}$ of the relevant sequencing primer. The COI amplicons were sequenced in one direction using the primer Aseq01F, and $28 \mathrm{~S}$ rDNA fragments were sequenced in two overlapping fragments using internal primers sy28SF11 (CATTTTCACTCTTCTCATGC) and sy28SR12 (AGCAAAGCATAGTACACATTTATA). Sequencing was performed with BigDye Terminator v3.1 on an ABI Prism 3130XL Analyzer (Applied Biosystems, Foster City, CA, USA). Sequence chromatograms were checked for accuracy and edited using FinchTV 1.3.1 (Geospiza, Seattle, WA, USA) and manually aligned in GenDoc v.2.7.000 (Nicholas and Nicholas 1997). All sequences have been deposited in GenBank under Accession Nos. as indicated in Table 1.

\section{RESULTS}

\section{Morphology}

Morphological analysis was carried out on the exoskeletons remaining after DNA extraction. All diagnostic morphological features of individuals were visible after treatment with the lysis buffer. Our results support the previous 

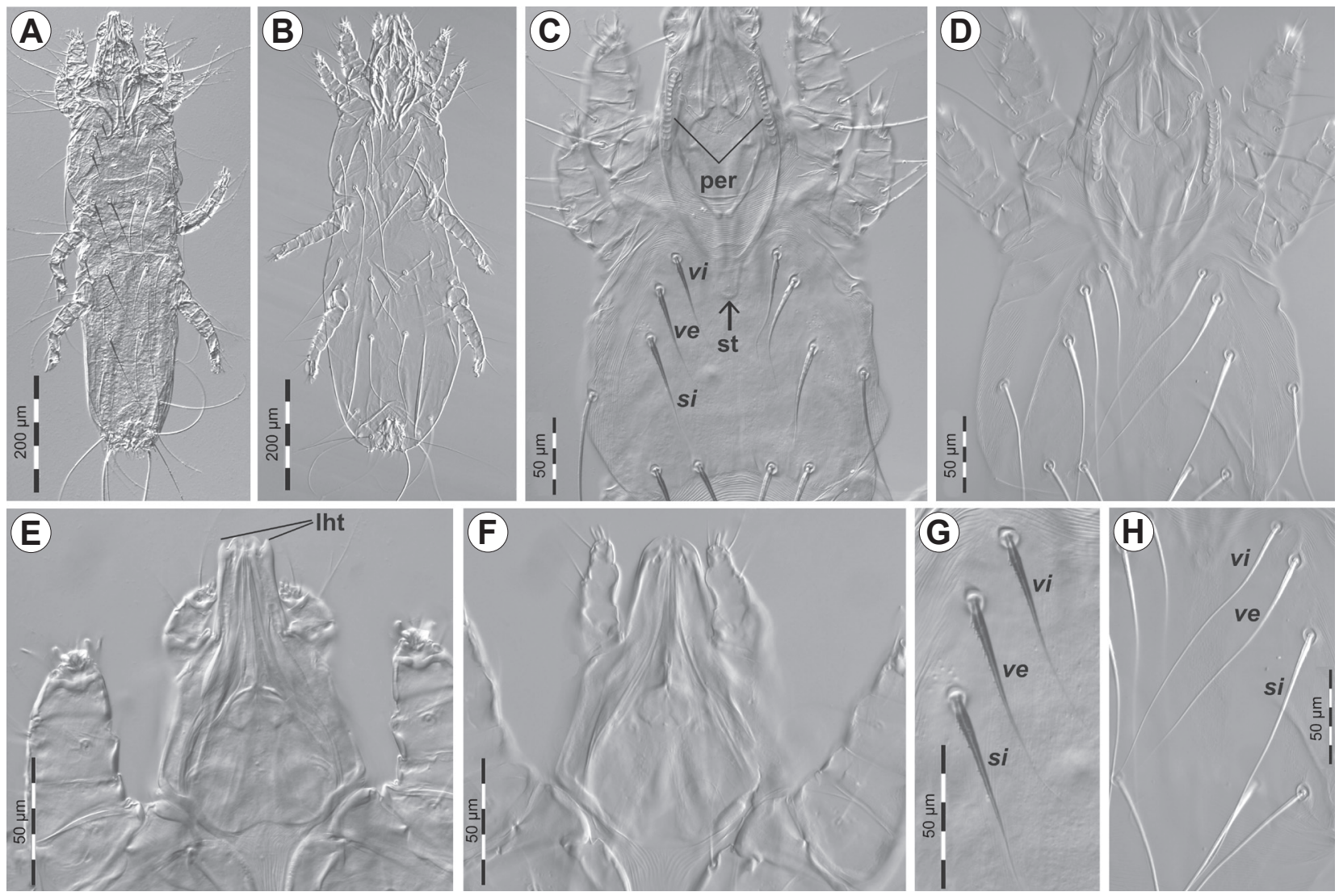

Fig. 1. Morphological characters of females of Stibarokris phoeniconaias (A, C, E, G) and Ciconichenophilus phoeniconaias (B, D, F, H). A, B - general view; C, D - gnathosoma and propodosoma; E, F - gnathosoma; G, H - propodonotal setae. Abbreviations: lht - lateral hypostomal teeth; per - peritremes; st - stylophore; vi, ve, si-propodonotal setae.

reported differences between females of S. phoeniconaias and C. phoeniconaias (Fig. 1A-H). In S. phoeniconaias, the female's body is strongly sclerotised (Fig. 1A), lateral hypostomal teeth are present (Fig. 1E), dorsal setae are strongly enlarged at the base, shorter, thick, and covered by spike-like structures (Fig. 1C,G). In C. phoeniconaias the female's body is weakly sclerotised (Fig. 1B), lateral hypostomal teeth are absent (Fig. 1F), dorsal setae (excluding terminal setae $f 1, f 2, h 1, h 2$ ) are longer (in the range of 1.5-2.5 times) and thinner than in S. phoeniconaias and are delicately knobbed (Fig. 1D,H).

\section{DNA barcoding}

We sequenced $616 \mathrm{bp}$ of the 5 -terminus of the COI gene and 1159 bp comprising D1-D3 region of 28S rDNA from two females representing $C$. phoeniconaias, three females and one male representing S. phoeniconaias, and one tritonymph, protonymph and larva (see Table 1 for details and GenBank Accession Nos.). All analysed sequences were identical and displayed the same COI haplotype, which indicates that all analysed specimens belong to one population of the same species. This result was supported by the analysis of the 28S rDNA gene fragment from nuclear DNA because all analysed specimens shared the same sequence in this variable gene fragment.

\section{DISCUSSION}

Our study based on mitochondrial and nuclear markers unambiguously supports conspecific status of Stibarokris phoeniconaias and Ciconichenophilus phoeniconaias. Although DNA-barcoding is not always sufficient ground for taxonomic decisions (its primary goal is accurate identification of known taxa), this tool strongly promotes our hypothesis in this particular case. The lack of any variation within the sequences suggests that all analysed specimens belong to the population founded by one female. Previous molecular studies on syringophilids have shown that the estimates of intraspecific genetic divergence between sequences representing COI and D2 region of the $28 \mathrm{~S}$ rDNA are $0.0-2.0 \%$ and $0.0-0.1 \%$, respectively for each markers (Glowska et al. 2013). Since the genus Ciconichenophilus and the species C. phoeniconaias were described later, the formal taxonomic consequence of our results is synonymization of $C$. phoeniconaias with S. phoeniconaias and Ciconichenophilus with Stibarokris.

The conspecific character of $S$. phoeniconaias and C. phoeniconaias reveals the existence of female dimorphism, a phenomenon never observed in syringophilids before. Among quill-inhabiting mites, there is only one other reported case of female polymorphism, in Pyonaca- 
rus aquilinus Dabert et Ehrnsberger, 1992 (Astigmata: Ascouracaridae) (Dabert and Ehrnsberger 1992), but its biological role remains unknown. The occurrence of dimorphic females in mites is usually associated with functional specialization of each morph, mainly with the presence of typical non-dispersing and modified dispersing forms. This phenomenon is often connected with phoresy, i.e. the association in which one organism (in this case a mite) is transported by another one (usually an insect) from place to place. Phoretic females have been observed in Mesostigmata, e.g. in Antennoseius janus Lindquist et Walter, 1989 and A. perseus Beaulieu, Déchêne et Walter, 2008 (Ascidae), in which they are associated with carabid beetles (Sericoda spp.). In these species the two female morphs differ mainly in the extent, shape and ornamentation of idiosomal shields, and the shape of dorsal setae (Lindquist and Walter 1989, Beaulieu et al. 2008). Female phoretomorphy has also been observed in several families of Prostigmata (the suborder to which syringophilids belong), in which dispersing females differ mainly in body sclerotisation, leg modifications and shape of gnathosoma (Cross and Moser 1975, Martin 1978, Magowski 1988, Walter and Proctor 1999).

With our current knowledge it is difficult to draw a final conclusion on the origin and biological role of dimorphic females in S. phoeniconaias. Since the differences between morphs (sclerotisation, setae length, thickness and ornamentation) are comparable to those characterizing migrating and sedentary individuals in other groups of mites, we hypothesise that we are dealing with a similar morph specialization in syringophilids. The nature of the differences indicates that the two morphs of females face different environmental pressures. Parameters such as humidity and temperature are stable and higher inside the quill than outside. Thus typical morphs (sedentary) are not exposed to drying and do not need to invest the energy in strong body sclerotisation to protect them against drying, and may expend energy on offspring production in a stable quill environment.

Mites hidden in the quill also do not have to deal with aerodynamic forces and thus may have long body setae to orient in the enclosed habitat. Migrating morphs are subjected to changing humidity and temperature and to wind; as a result, their dorsal setae have to be reduced to restrict the body surface area (Proctor 2003). Moreover, setae provided with spike-like structures allow females to attach to the barbules. The presence of lateral hypostomal teeth (seen in 'armored morph'; Fig. 1A,E) is absolutely unique feature in syringophilid quill mites (observed only in species of Stibarokris). The shape and position of these sclerites suggest they role in embracing the quill from the outside during cutting it open with chelicerae or being the points of support during drilling holes to enter new quills.

Another question arises if we assume that females of S. phoeniconaias are functionally divided in two morphs, reproductive (never leaving the quill) and those which move from quill to quill and horizontally disperse between adult birds: why this phenomenon has not been previously noted in other species? In our opinion, the biology of $S$. phoeniconaias should not be treated by analogy to the model species Syringophiloidus minor (Berlese, 1887) (Kethley 1971, Casto 1974a-d, 1975a-c, 1976) because of biological differences between host species, the American flamingo and the house sparrow, which are crucial for mite dispersion. In flamingo, first horizontal transfer of mites from parents to descendants should be delayed because of longer maturation. Also dispersion associated with molting should occur in different way, because of irregular molting. It seems to be more cost effective to stay in long-lived flamingo individual, colonise and exploit possibly wide spectrum of its more numerous and less frequently lost feathers compared to sparrow, than just transfer on offspring. Since flamingos live in large colonies, including thousands of individuals, it is very likely that transfer between adult birds also occurs, which would be more economic spreading than merely vertical transmission (only one descendant per year).

At the present state of knowledge of syringophilid biology, including dispersal, we can only guess at the role of the two female morphs of $S$. phoeniconaias. Future studies should focus on details of dispersal strategy and life cycle of this species, including determination of the founder morph type and examination of the genetic structure of population, which could provide information on how many female founders colonise a quill. Without empirical research both on host and mite species we will remain far from understanding the female dimorphism phenomenon in this species.

Acknowledgements. We are very grateful to Dr. H. Dastych from the Biozentrum Grindel und Zoologisches Museum Hamburg in the University of Hamburg, Germany for the help in the collection of the material. This study was supported by the Polish Ministry of Science and Higher Education (grant no. NN303 561 939).

\section{REFERENCES}

Beaulieu F., Déchêne A.D., Walter D.E. 2008: Phase morphs and phoresy: New species of Antennoseius (Vitzthumia) mites (Acari: Mesostigmata: Ascidae) associated with pyrophilous carabids (Carabidae: Sericoda spp.) in Alberta, Canada. Zootaxa 1961: 37-57.
Casto S.D. 1974a: Quill wall thickness and feeding of Syringophiloidus minor (Berlese) (Acarina: Syringophilidae). Ann. Entomol. Soc. Am. 67: 824. 
CASTO S.D. 1974b: Entry and exit of syringophilid mites (Acarina: Syringophilidae) from the lumen of the quill. Wilson Bull. 86: 272-278.

CAsto S.D. 1974c: A nocturnal dispersal rhythm in the quill mite, Syringophiloidus minor (Berlese) (Prostigmata: Syringophilidae). J. Med. Entomol. 11: 113-114.

CASTO S.D. 1974d: Observations on the karyotype and maturation of the F1 generation of Syringophiloidus minor (Acarina: Syringophilidae). Ann. Entomol. Soc. Am. 67: 136-137.

Casto S.D. 1975a: Generation time of male quill mites Syringophiloidus minor (Berlese) (Acarina: Syringophilidae). Am. Midl. Nat. 93: 495-498.

Casto S.D. 1975b: The effect of the postjuvenal molt in the House Sparrow on infestations of the quill mite, Syringophiloidus minor (Berlese) (Acarina: Syringophilidae). J. Med. Entomol. 12: 23-27.

CAsto S.D. 1975c: Mortality in the quill mite, Syringophiloidus minor (Acarina: Syringophilidae). Ann. Entomol. Soc. Am. 68: $551-552$

CASTO S.D. 1976: Host records and observations of quill mites (Acarina: Syringophilidae) from Texas birds. Southwest. Entomol. 1: $155-160$.

Clements J.F. 2007: The Clements Checklist of Birds of the World. Sixth Edition, Cornell University Press, Ithaca, New York, $864 \mathrm{pp}$.

Cross E.A., Moser J.S. 1975: A new, dimorphic species of Pyemotes and a key to previously-described forms (Acarina: Tarsonemoidea). Ann. Entomol. Soc. Am. 68: 723-732.

Dabert M., Bigos A., Witalinski W. 2011: DNA barcoding reveals andropolymorphism in Aclerogamasus species (Acari: Parasitidae). Zootaxa 3015: 13-20.

Dabert J., Ehrnsberger R. 1992: Neue Arten bei der Federmilbenfamilie Ascouracaridae Gaud \& Atyeo, 1976. Osnab. Naturwissen. Mitt. 18: 109-150.

Dabert J., Ehrnsberger R., Dabert M. 2008: Glaucalges tytonis sp. n. (Analgoidea, Xolalgidae) from the barn owl Tyto alba (Strigiformes, Tytonidae): compiling morphology with DNA barcode data for taxon descriptions in mites (Acari). Zootaxa 1719: 41-52.

Glowska E., Dragun-Damian A., Dabert J. 2012a: Picobia $d z$ iabaszewskii sp. nov. (Acari, Syringophilidae) - combined description (morphology with DNA barcode data) of a new quill mite species parasitizing Garrulax formosus (Passeriformes: Leiothrichidae). Zootaxa 3224: 57-61.

Glowska E., Dragun-Damian A., Dabert J. 2012b: A new quill mite Syringophiloidus pseudonigritae sp. nov. (Prostigmata, Syringophilidae) parasitizing Pseudonigrita arnaudi (Passeriformes, Ploceidae) - a combined description using morphology and DNA barcode data. Zootaxa 3532: 64-68.

Glowska E., Dragun-Damian A., Dabert J. 2013: DNA-barcoding contradicts morphology in quill mite species Torotrogla merulae and T. rubeculi (Prostigmata: Syringophilidae). Folia Parasitol. 60: 51-60.

Glowska E., Skoracki M. 2011a: Two new quill mite species (Acari, Cheyletoidea, Syringophilidae) parasitizing Dinemellia

Received 16 December 2013 dinemelli (Rüppell) (Passeriformes, Ploceidae). Zootaxa 3114: 63-68.

Glowska E., Skoracki M. 2011b: New species of quill mites (Acari, Cheyletoidea, Syringophilidae) and the first record of male for the genus Stibarokris. Zootaxa 2817: 63-68.

Kethley J.B. 1971: Population regulation in quill mites (Acari: Syringophilidae). Ecology 52: 1113-1118.

Lindquist E.E., Walter D.E. 1989: Antennoseius (Vitzthumia) janus n. sp. (Acari: Ascidae), a mesostigmatic mite exhibiting adult female dimorphism. Can. J. Zool. 67: 1291-1310.

Magowski W.Ł. 1988: Description of a new species of Formicomotes Sevastianov, 1980 (Acari: Dolichocybidae) with notes on the female dimorphism within this genus. Mitt. Hamb. Zool. Mus. Inst. 85: 163-182.

Manson D.C.M., Oldfield G.N. 1996: Life forms, deutogyny, diapause and seasonal development. In: E.E. Lindquist, M.W. Sabelis and J. Bruin (Eds.), Eriophyoid Mites: Their Biology, Natural Enemies and Control. Elsevier, Amsterdam, pp. 173 183.

Martin N.A. 1978: Siteroptes (Siteroptoides) species with Pediculaster-like phoretomorphs (Acari: Tarsonemida: Pygmephoridae) from New Zealand and Polynesia. N.Z. J. Zool. 5: 121-155.

Mironov S.V., Dabert J., Dabert M. 2012: A new feather mite species of the genus Proctophyllodes Robin, 1877 (Astigmata, Proctophyllodidae) from the long-tailed tit Aegithalos caudatus (Passeriformes: Aegithalidae) - morphological description with DNA barcode data. Zootaxa 3253: 54-61.

Nicholas K.B., Nicholas H.B. Jr. 1997: GeneDoc: a tool for editing and annotating multiple sequence alignments. Pittsburgh Supercomputing Center's National Resource for Biomedical Supercomputing, ver. 2.7.000. http://www.nrbsc.org/downloads (accessed 2 March 2007).

Proctor H.C. 2003: Feather mites (Acari: Astigmata): ecology, behavior, and evolution. Annu. Rev. Entomol. 48: 185-209.

Proctor H.C., Williams G., Clayton D.H. 2009: Population density and male polymorphism in the feather mite Falculifer rostratus (Acari: Falculiferidae). In: M.W. Sabelis and J. Bruin (Eds.), Trends in Acarology - Proceedings of the 12th International Congress of Acarology, 21.-26. August 2006. Springer, Amsterdam, pp. 299-302.

RADWAN J. 2001: Male morph determination in Rhizoglyphus echinopus (Acaridae) Exp. Appl. Acarol. 25: 143-149.

Regev S. 1974: Morphological and genetic evaluation of male polymorphism in Cheyletus malaccensis (Cheyletidae: Acarina). Acarologia 16: 85-93.

SkoRACKi M. 2011: Quill mites (Acari: Syringophilidae) of the Palaearctic region. Zootaxa, Suppl. 2840, 416 pp.

Skoracki M., OConNor B. 2010: New taxa of quill mites (Acari: Cheyletoidea: Syringophilidae). Zootaxa 2341: 1-32.

Volgin V.I. 1969: Acarina of the Family Cheyletidae of the World. Translation from Russian (1987). Amerind Publishing Co., New Delhi, 532 pp.

Walter D.E., Proctor H.C. 1999: Mites: Ecology, Evolution and Behaviour. University of NSW Press, Sydney and CABI, Wallingford, $322 \mathrm{pp}$

Accepted 25 January 2014 